\title{
ROTATIONAL LORDOSIS: THE DEVELOPMENT OF THE SINGLE GURVE
}

\author{
E. W. Somerville, Oxford, England \\ From the Nuffield Orthopaedic Centre, Oxford
}

A review of the literature on curvature of the spine-with particular reference to the deformity that has come to be called scoliosis-cannot but fill the reader with a sense of frustration. Despite the recording of a mass of evidence-much of it conflicting - and the presentation of many different explanations, the commonest form of the deformity is still rightly called "idiopathic." The problem is clearly one that should not be approached lightly, and it is with some trepidation that a further hypothesis is suggested in a paper which at present can be little more than a preliminary report.

A hundred years ago scoliosis was much discussed, and Sir William Adams, in his lectures published in 1864, summed up the more authoritative views of the time when he said that: "Rotation plus lordosis equals lateral flexion"; but the inference throughout was that rotation was the primary factor. It seems more logical and less misleading to describe deformities of the spine in relation to the spine itself as a unit rather than to describe them in relation to the body as a whole. To describe rotation plus lordosis as a scoliosis can be nothing but misleading, for scoliosis is defined as lateral flexion of the spine. This then is my excuse for suggesting a new concept and a new name for an old disease.

The deformity-Figure 1 shows a spine with a considerable curvature of the type commonly called structural scoliosis. If we examine the curve critically we see that at the apex the vertebrae are almost in lateral view, with their bodies on the convexity and the neural arches in the concavity. This curve is therefore a lordosis. That rotation is present is obvious. Unfortunately the deformity is not quite so simple as this. In the early stages, when only minimal deformity is present, the lateral view shows a small but definite mid-thoracic lordosis (Fig. 2). As rotation increases the spine becomes flattened (Fig. 3). Later an apparent kyphosis develops (Fig. 4), and increases (Fig. 5). By this time there is 70 degrees or more of rotation, so that what is seen as kyphosis is in fact

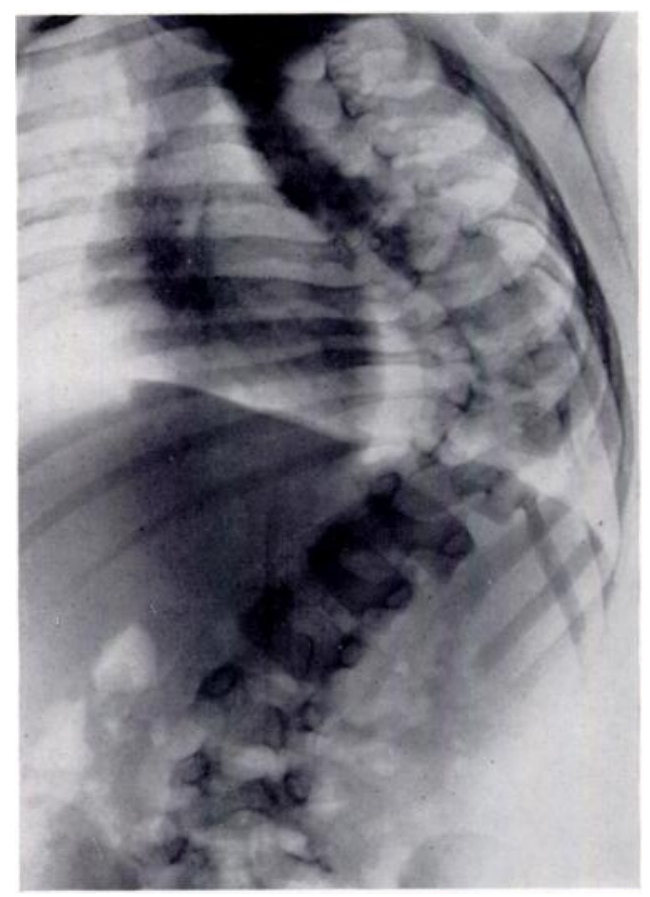

FIG. 1

Typical " structural scoliosis." lateral flexion of the spine. Analysed, the deformity consists of lordosis, rotation and lateral flexion. It is the purpose of this paper to describe a mechanism by which such a deformity may develop and to indicate some ways in which the local mechanics mar influence the final curve. 


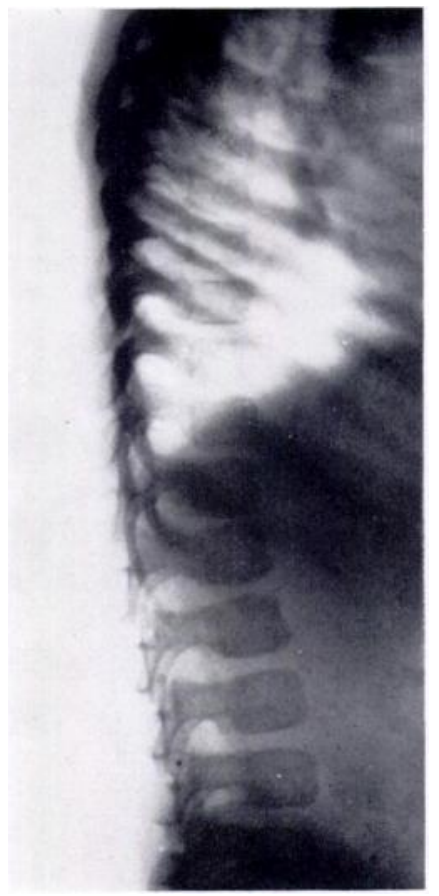

FIG. 2
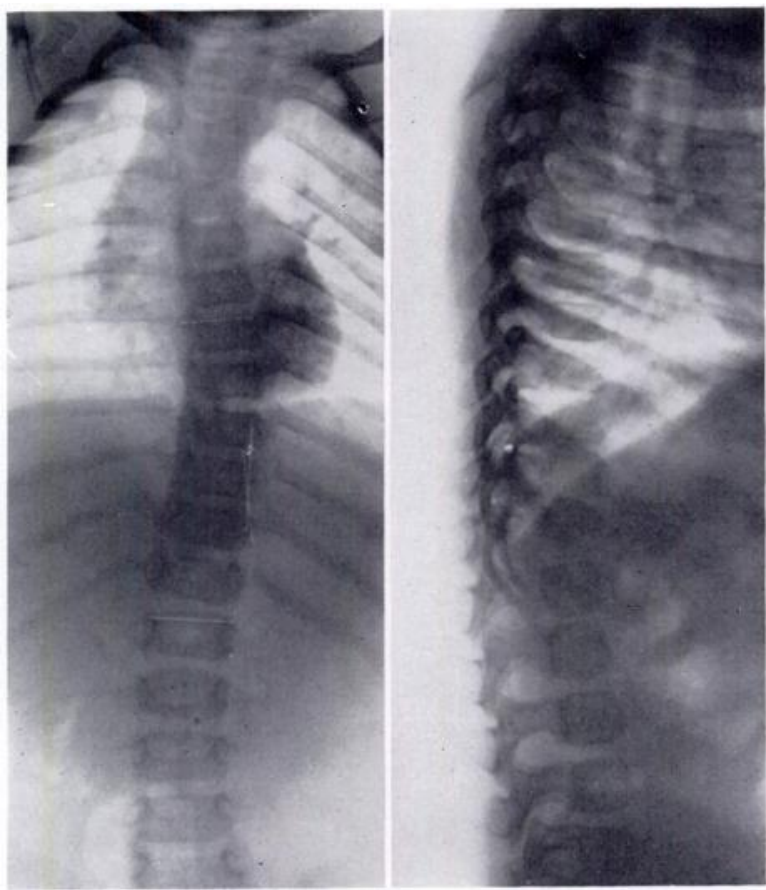

FIG. 3

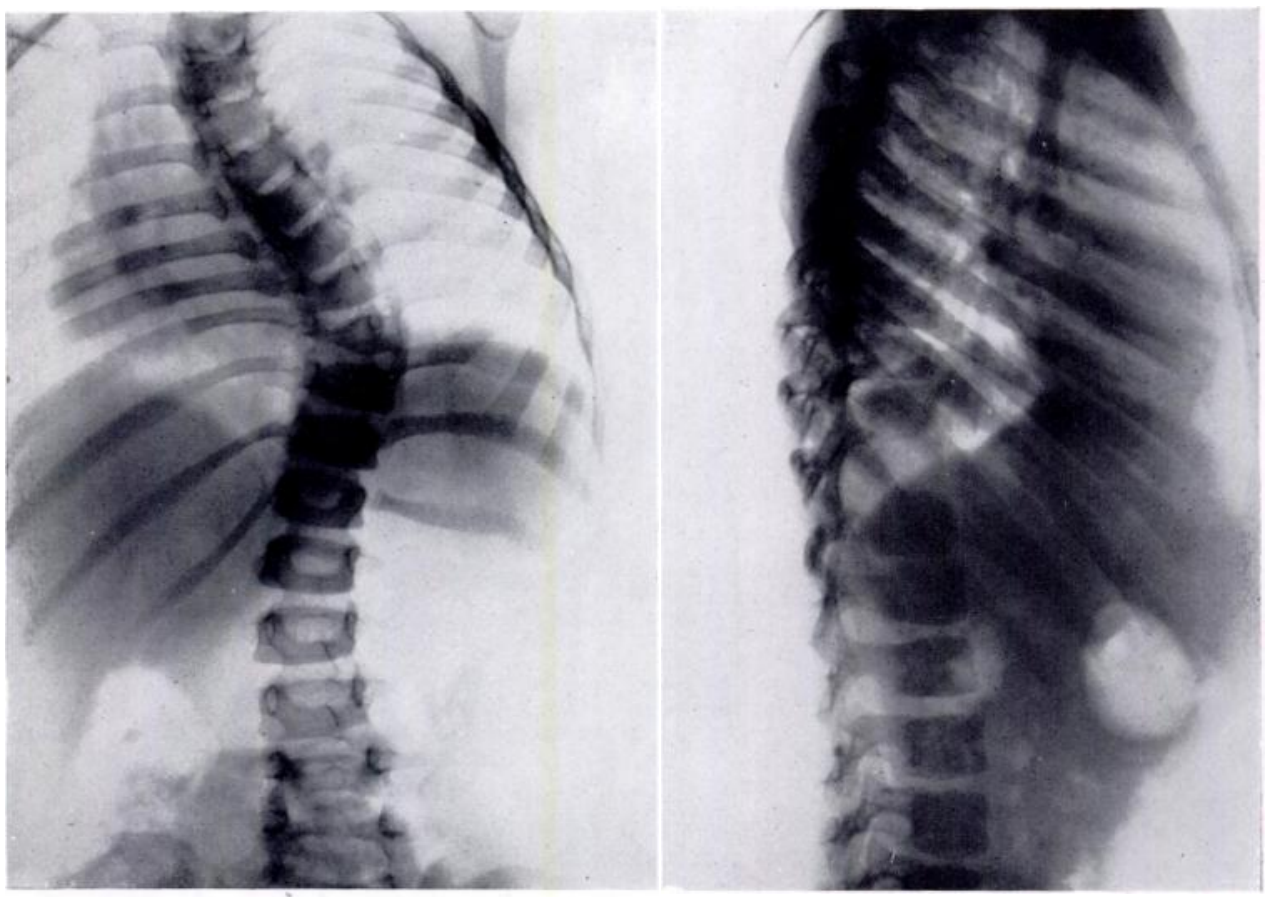

FIG. 4

Stages in the progressive development of the deformity. 


\section{PRODUCTION OF THE DEFORMITY}

The primary mechanism - It is probably easier to appreciate the deformity if the sequence of its development is studied.

Lordosis-The simplest way in which a localised structural lordosis could develop would be by a failure of growth of the posterior elements of the vertebrae (by this is meant all that part of a vertebra lying behind the body). If this were to happen to an exaggerated extent it would be possible for a localised lordosis of 90 degrees or more to develop. This is a deformity that we seldom recognise because, as it develops, compensatory changes occur in the spine and the original deformity is rapidly obscured.

Compensation-To compensate a slowly developing structural lordosis the spine is flexed forwards. At first a small kyphos develops above and below (Fig. 3). But soon it is impossible for further forward flexion to occur without bone changes developing and it is easier for

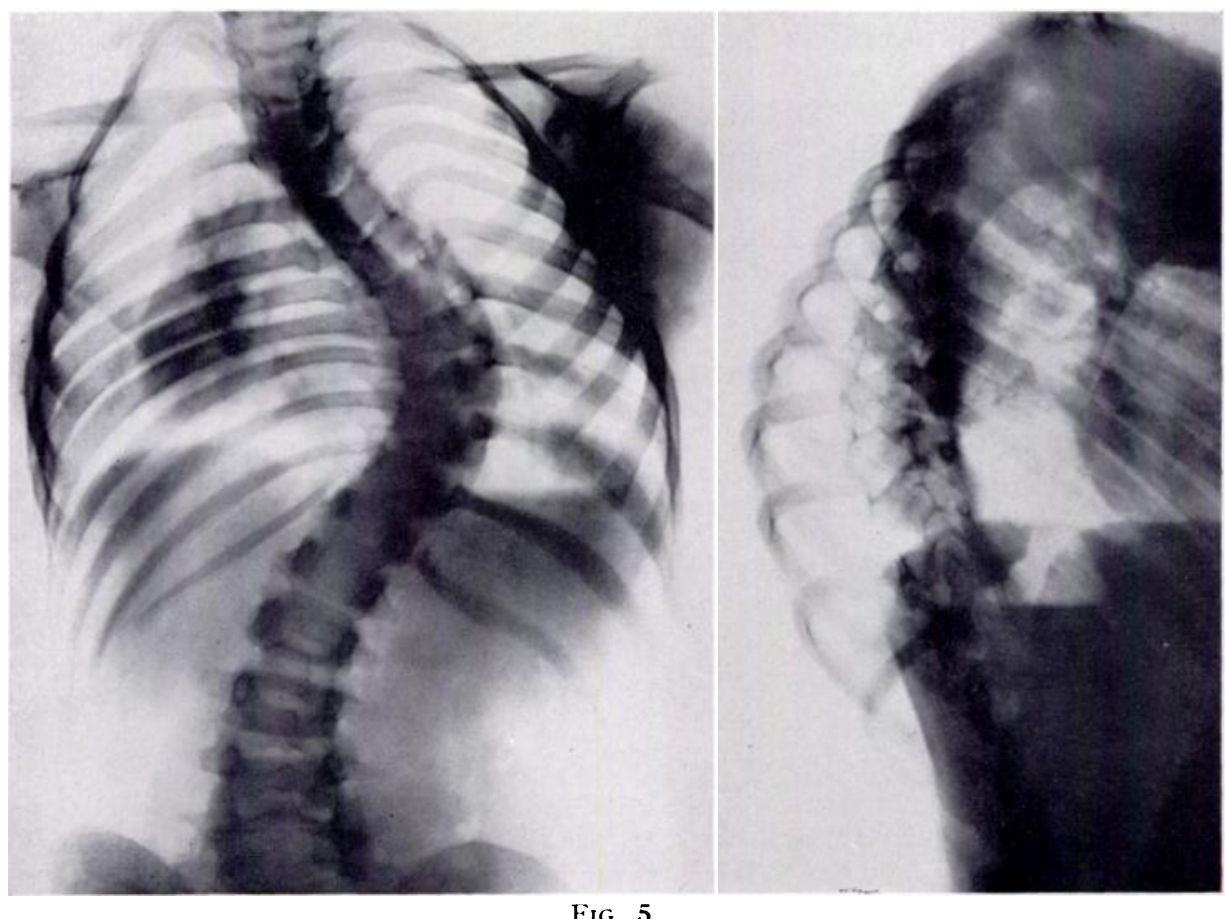

The fully established deformity.

the affected area of the spine to rotate instead; then simple lateral flexion of the normal parts of the spine above and below will bring the head once more above the pelvis and result in an appearance very like that seen in Figure 1.

Rotation-It is easier to demonstrate the mechanism of this than to describe it. Figure 6 shows a model which represents a spine. The vertebral bodies are represented by wood blocks separated by "intervertebral discs" of sponge rubber. Hooks in the backs of the "vertebral bodies" represent the posterior elements, and these are joined together in such a way that the upper and lower parts are relatively straight but in the middle they are joined more tightly so that there is a small " lordosis." For the purposes of the experiment we must assume that the lordosis has already developed. If the model is flexed forwards in an attempt to overcome the lordosis, at first a kyphosis develops above and below, and then the affected area begins to rotate (Fig. 7). The more it is flexed the more it rotates, and if the two ends are approximated to represent the effect of gravity the rotation is still further increased (Fig. 8). Distraction produces the reverse effect.

vol. $34 \mathrm{~B}$, No. 3, AUGUST 1952 
Assessment of rotation-It is of value to be able to make an estimate of the degree of rotation of the vertebrae involved in a curve. This may be done by making a standard for comparison by radiographing a vertebra in different positions of rotation. This sometimes shows a remarkable degree of rotation between two adjacent vertebrae. For example, a study of the radiograph shown in Figure 1 indicates that T.12 is rotated at least 60 degrees, whereas L.1 is rotated only 10 degrees.

Lateral flexion-It is not intended here to discuss the mechanics of the different parts of the spine: suffice it to say that rotation is easy at first but meets with increasing resistance which entails moulding of bone. On the other hand, as rotation increases lateral flexion of the spine itself (that is, forward flexion in relation to the body) meets with less resistance so that a point is reached at which the resistance to each is the same. From this point onwards considerable pressure is applied to one side of the vertebrae. At first this is absorbed by the

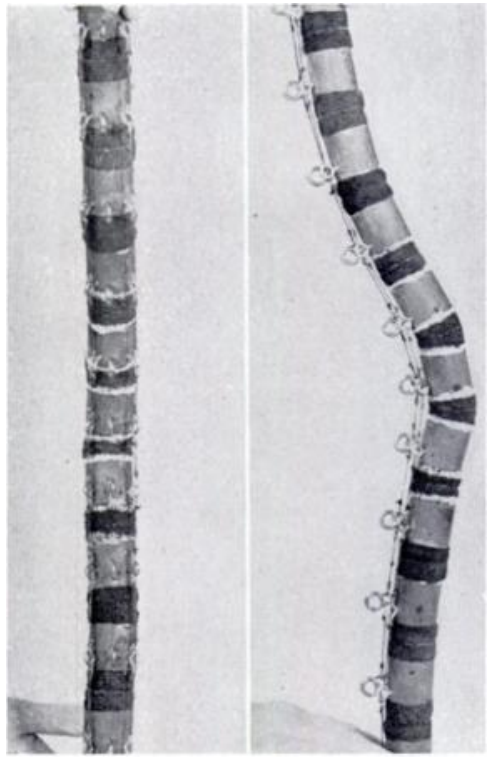

FIG. 6

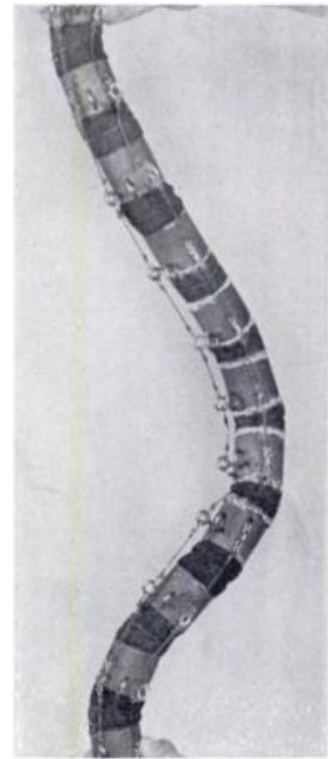

FIG. 7

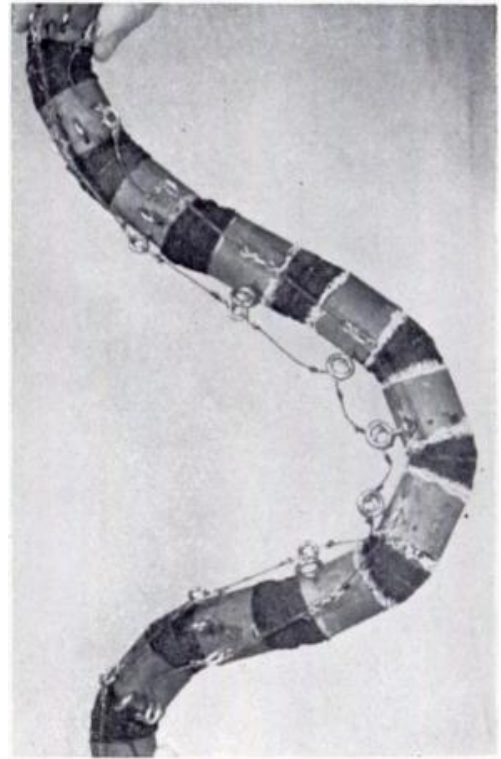

Fig. 8

Figure 6-The model shows the localised fixed lordosis. Figure 7-Rotation produced by forward flexion. Figure 8-Rotation increased by compression such as might resemble that due to gravity.

resilience of the intervertebral discs, but soon the pressure falls on the soft growing bone of the vertebral bodies so that their growth on one side is depressed and they become wedged, with further increase of the deformity.

The secondary mechanism-There is one other factor which should be added to the above mechanism. After a certain amount of lordosis has developed the posterior articulations rapidly lose their flexibility and become increasingly rigid. We then have a "spine" in which the posterior elements will not bend and do not grow. If under these circumstances the vertebral bodies continue to grow they can only do so by being displaced laterally, that is, by increasing the degree of rotation. It is not always possible to say just how much each of these mechanisms plays a part in the production of any one curve, but it seems probable that both are in operation at the same time, the former decreasing in influence, the latter increasing.

\section{AN EXPERIMENTAL STUDY}

So far I have given only a theoretical concept of the way in which this deformity may develop. In an experimental study on young rabbits, an attempt was made to prevent growth 
of the neural arch by cauterisation of the laminae on each side of the spinous processes. In the first experiments this did not cause a recognisable deformity because the posterior ligaments were destroyed and the laminae and facets merely separated as the bodies grew. The ligaments were therefore reinforced by wire loops lightly placed round the spinous processes before the laminae were burned. In the three surviving rabbits in which the operation was done on four adjacent vertebrae a curve has developed which closely resembles that seen in man, including rotation and deformity of the ribs (Figs. 9 to 13). Deformity was first noticed three weeks after operation.

Three points about these specimens should be mentioned. 1) Much experimental work has been done by others in an attempt to produce curvature of the spine. Usually these experiments have been based on attempts to arrest growth in one side of adjacent vertebral bodies. This has resulted in simple lateral flexion of the spine but, as far as can be judged from examination of the illustrations, there has been little or no rotation. In our experiments considerable

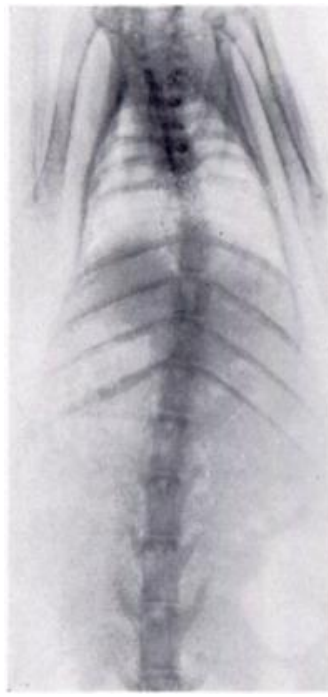

FIG. 9

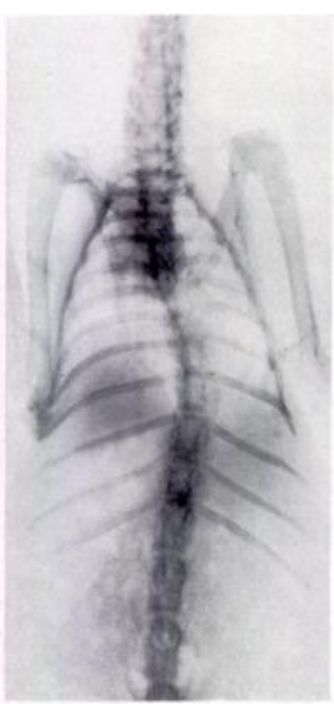

FIG. 10

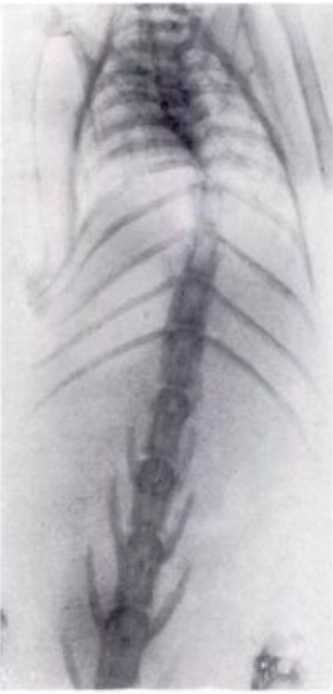

FIG. 11

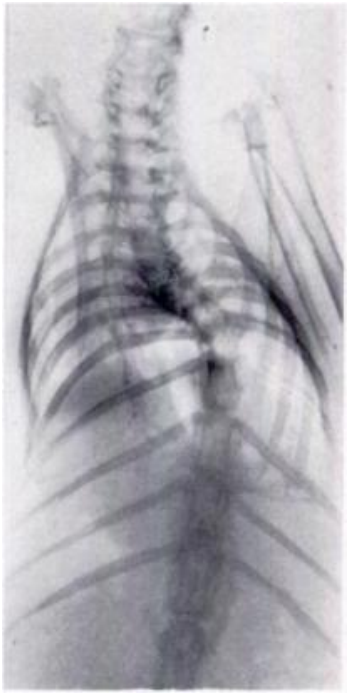

FIG. 12

Development of a thoracic curve produced artificially in a rabbit. Serial radiographs taken at five-weekly intervals.

rotational deformity developed. 2) The deformity in our experiments has been produced in an animal in which it is unknown in nature and in which gravity can play no part. 3) All the curves have gone to the right although every effort was made to apply heat equally to both sides. The number is, of course, small and this may be coincidence.

When three vertebrae were operated on the curve developed much more slowly, and was not visible before seven weeks from the date of operation in the only rabbit which survived long enough. When one or two vertebrae have been treated and the animal has survived, no curve has been produced so far. But it is too soon to draw conclusions, and a curve may yet be produced by operating upon a smaller number of vertebrae. It must be remembered that experiments such as those described are very rough compared with the refinements of nature.

Controls have been done which cover some of the possible errors. 1) The laminae have been burnt, without wiring of the spinous processes. 2) The spinous processes have been wired together, without burning the laminae. 3) The muscles on both sides have been stripped from the laminae and lightly burnt. 4) The muscles on one side only have been stripped from the laminae. In none of these animals has any curve resulted.

vol. 34 B, xo. 3, AUgust 1952 


\section{FACTORS AFFECTING THE TYPE OF CURVE}

There are two chief factors which will determine the type of curve, namely, different mechanics in different parts of the spine, and variations in the extent of growth changes.

Mechanics of the spine-The relevant part of the spine is divided anatomically into two sections. In the thoracic spine the articular facets lie on a circle, the centre of which is within or just in front of the intervertebral disc, so rotation is free. In the lumbar spine the articular facets lie on a circle whose centre is posterior to the facets; consequently rotation is possible only to the limit of the looseness of the ligaments and to the amount that one vertebral body can be displaced laterally on its neighbours. This allows only a few degrees of movement without stretching of ligaments or moulding of bone. The other important difference between the two segments is the attachment of the ribs to the thoracic vertebrae. The ribs do not seriously affect the mechanics of normal rotation, but localised

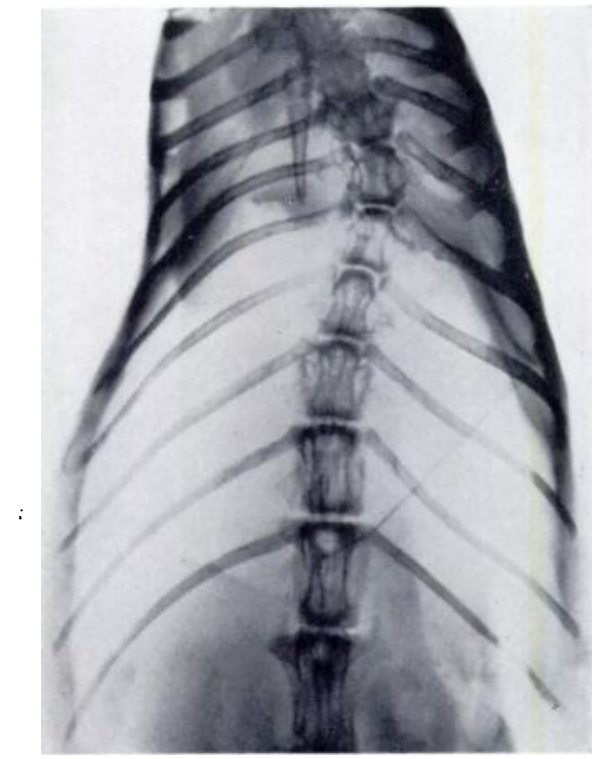

FIG. 13

Radiograph of the spine of another rabbit taken post-mortem.

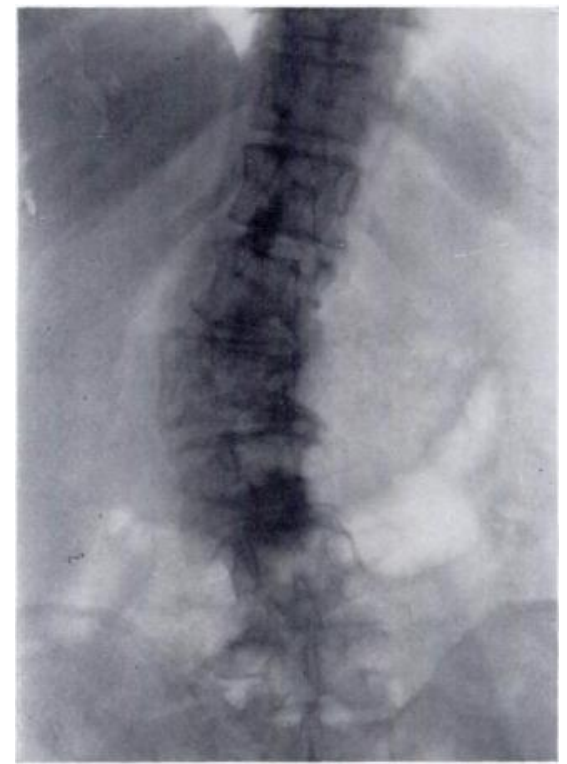

FIG. 14

Lumbar scoliosis in which subluxation of the vertebral bodies gradually occurred.

rotation can occur only if the ribs are moulded, often to an extreme degree. Thus rotation in the thoracic spine is restricted by the ribs, and in the lumbar spine by the shape and position of the articular facets. This difference of rotation exerts the greatest influence on the character of the ultimate curve. In rotational lordosis of the spine rotation is produced by considerable force applied to the spine in an attempt to compensate the lordosis. If this force is applied to regions of a spine in which different mechanics exist, the same forces will produce different curves in the different segments. For example, we find that a thoracic curve is quite unlike a lumbar curve. In the thoracic region the structural changes that occur are either in the ribs or in the vertebral bodies rather than in joints, so that deformity is severe and pain slight. In the lumbar spine the reverse is the case. Extensive rotation can take place only by subluxation of one vertebral body on the next (Fig. 14) and by subluxation and moulding of the articular processes; deformity is less severe but pain is a noticeable feature.

Variations in the growth defect-There is no certain way of determining how many vertebrae are primarily involved, but examination of many radiographs suggests that the 
number may vary considerably. The appearances also suggest that all the vertebrae primarily affected may not be involved to an equal extent. Thus there are many possible variations, about which our knowledge is at present scanty, but which may play a big part in decompensation.

\section{DISCUSSION}

A hypothesis has been presented that a complex deformity consisting of lordosis, rotation and lateral flexion-which by custom is called structural scoliosis-is the result of a chain of events initiated by a structural lordosis arising from failure of growth of the posterior elements of a segment of the spine. There is no difficulty in postulating this hypothesis in certain congenital abnormalities, nor in the complicated curves of cretins with their patchy growth disturbance, and for a long time it has been appreciated that growth plays an important part in the development of idiopathic structural scoliosis, though we may not know the cause of the growth disturbance. But we find the most excellent examples of this deformity in certain cases of anterior poliomyelitis. Deformity after poliomyelitis is usually attributed to muscle imbalance, but it may be difficult to say where the imbalance lies and sometimes the curve seems to go in an unexpected direction! There are many similarities between the curve in anterior poliomyelitis and the curve found in such conditions as idiopathic structural scoliosis, and the only real difference is that the curve in poliomyelitis is usually longer. A feature of poliomyelitis is the way in which the growth of a bone closely associated with paralysed muscles may be interfered with to a greater or lesser extent for a longer or shorter time. In the relevant area of the spine the only part of the vertebrae to which muscles have important attachments are the various parts of the neural arch, and if its growth were disturbed as a result of paralysis of the sacrospinalis muscle a rotational lordosis would result. It has been mentioned already that a variable number of vertebrae may be primarily involved and it seems unlikely that in poliomyelitis less than five or six would be affected, and often many more. This would result in a long curve. In the idiopathic deformity it is more likely that a smaller number of vertebrae are involved, resulting in a tighter, shorter and more vicious-looking deformity.

\section{SUMMARY AND CONCLUSIONS}

This paper is a theoretical and experimental study of the processes involved in the development of curvature of the spine which we have come to call scoliosis. Reasons are advanced why the term scoliosis in its modern meaning may be misleading and an alternative title of Rotational Lordosis is suggested. It is admitted that it is more cumbersome, but I maintain that it is more accurately descriptive of the deformity under discussion and differentiates it from other curvatures which are produced differently but at present all of which come under the heading of scoliosis. I am conscious that many gaps still remain unfilled, especially the important but unexplained problem of the nature of the growth disturbance. If we could solve this we would be near to a solution of one of the most bizarre, mysterious and crippling deformities with which we are faced.

I wish to thank my colleagues at the Nuffield Orthopaedic Centre for their help. I am especially grateful to Professor J. Trueta for placing at my disposal facilities for carrying out the experiments and for much helpful advice whenever needed, and to $\mathrm{Mr}$ J. C. Scott for access to his extensively documented and illustrated cases collected and analysed over many years.

\section{REFERENCE}

Adams, W. (1865): Lectures on the Pathology and Treatment of Lateral and Other Forms of Curvature of the Spine. London: J. Churchill and Sons.

vol. 34 B, No. 3, AUgust 1952 